\title{
OPTIMAL REGULARITY OF THE CONVEX ENVELOPE
}

\author{
GUIDO DE PHILIPPIS AND ALESSIO FIGALLI
}

ABSTRACT. We prove sharp regularity results for the convex envelope of a continuous function inside a convex domain.

\section{INTRODUCTION}

Given an open set $\Omega \subset \mathbb{R}^{n}$ and a continuous function $v: \bar{\Omega} \rightarrow \mathbb{R}$, we define its convex envelope in $\Omega$ as

$$
\Gamma_{v}(x):=\sup \{\ell(x): \ell \leq v \text { in } \bar{\Omega}, \ell \text { affine }\} .
$$

We immediately see that $\Gamma_{v}: \bar{\Omega} \rightarrow \mathbb{R}$ is the largest convex function below $v$ in $\Omega$.

The issue of the regularity of the convex envelope (or inf-sup convolution) appears in several problems from analysis and geometry. To mention some: the AlexandrovBakelman-Pucci estimate for fully non-linear equations 4, 9, the Monge-Ampère equation [3, 7, the study of geometric flows [6, 12, the Hamilton-Jacobi equation [2,8], etc. Moreover, as observed in [13, the convex envelope can also be seen as a solution of a non-linear obstacle problem.

Since convex functions are locally Lipschitz, one wants to understand under which assumptions the convex envelope is of class $C^{1}$ or better. Several results have been established in this direction [5, 10, 11, 14, but they all deal either with the Dirichlet problem (i.e., $\Gamma_{v}$ is the convex envelope of $\left.v\right|_{\partial \Omega}$ ), or they make some suitable global assumptions on $v$ in order to "avoid" the influence of the boundary (see for instance Theorem 4.1 below). Our goal here is to investigate the regularity of $\Gamma_{v}$ in full generality.

Before stating our results, we recall the following definition:

Definition 1.1. Given $\alpha \in(0,1]$, a continuous function $v$ is said to be $(1+\alpha)$ semiconcave in $\bar{\Omega}$ if for every $x_{0} \in \bar{\Omega}$ there exists a slope $p_{x_{0}} \in \mathbb{R}^{n}$ such that

$$
v(x) \leq v\left(x_{0}\right)+p_{x_{0}} \cdot\left(x-x_{0}\right)+C\left|x-x_{0}\right|^{1+\alpha} \quad \text { for every } x \in \bar{\Omega} \cap B\left(x_{0}, \varrho_{0}\right)
$$

for some constants $C$ and $\varrho_{0}$ independent of $x_{0}$.

The following two theorems relate the regularity of $\Gamma_{v}$ to the one of $v$ and $\Omega$.

Theorem 1.2. Let $\alpha, \beta \in(0,1], \Omega$ be a bounded convex domain of class $C^{1, \beta}$, and $v: \bar{\Omega} \rightarrow \mathbb{R}$ be a globally Lipschitz function which is $(1+\alpha)$-semiconcave in $\bar{\Omega}$. Then $\Gamma_{v} \in C_{\mathrm{loc}}^{1, \min \{\alpha, \beta\}}(\Omega)$.

Theorem 1.3. Let $\Omega$ be a bounded uniformly convex domain of class $C^{3,1}$, and let $v \in C^{3,1}(\bar{\Omega})$. Then $\Gamma_{v} \in C^{1,1}(\bar{\Omega})$.

Received by the editors January 31, 2013 and, in revised form, February 20, 2013 and October 17, 2013.

2010 Mathematics Subject Classification. Primary 49J52. 


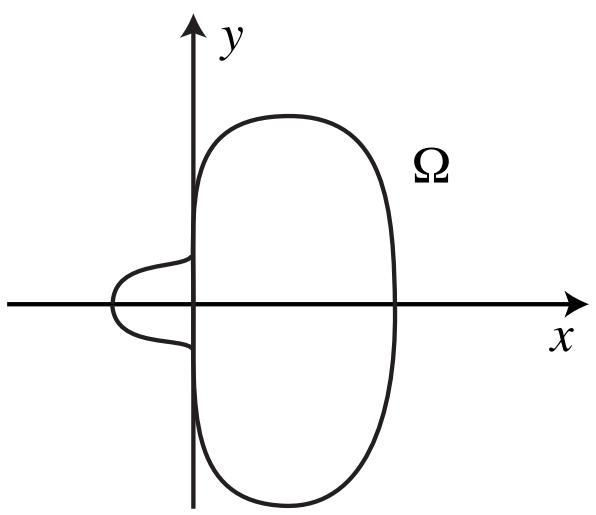

Figure 2.1. If $\Omega$ is a $C^{\infty}$ domain as above, then the function $|x|$ is of class $C^{\infty}$ on $\partial \Omega$. This situation illustrates that convexity of $\Omega$ is necessary for regularity.

As we will show in the next section, all assumptions made in the above theorems are sharp.

The paper is structured as follows: in Section 2 we provide some examples showing that our results are optimal. Then in Section 3 we recall some preliminaries about convex sets and functions, and we prove some criteria to show that a function is $C^{1, \alpha}$. Sections 4 and 5 are devoted to the proof of Theorem 1.2 and Theorem 1.3. respectively. Moreover, at the end of Section 4 we also discuss some extension/variant of Theorem 1.2 .

\section{Counterexamples}

Here we present some counterexamples showing the sharpness of our assumptions in Theorems 1.2 and 1.3 . The construction of the counterexamples are mainly based on the following observation: If $v: \bar{\Omega} \rightarrow \mathbb{R}$ is harmonic in $\Omega$, then (by the maximum principle)

$$
\Gamma_{v}=\sup \{\ell: \ell \leq v \text { in } \partial \Omega, \ell \text { affine }\} .
$$

All counterexamples are done in $\mathbb{R}^{2}$, and to simplify the notation, only for this section we will use $(x, y)$ to denote the coordinates of a point in $\mathbb{R}^{2}$ (starting from the next section, $x$ and $y$ will be used to denote generic points in $\mathbb{R}^{n}$ ).

The set $\Omega$ has to be convex. Let $\Omega$ be a $C^{\infty}$ domain as in Figure 2.1, with a flat part along the $y$ axis. Then the function $|x|$ is of class $C^{\infty}$ when restricted to $\partial \Omega$. (Indeed, if $x=f(y)$ locally parameterizes $\partial \Omega$ near the axis $\{x=0\}$, then $y \mapsto|x|=|f(y)| \in C^{\infty}$.) So, by elliptic regularity, its harmonic extension $v$ inside $\Omega$ belongs to $C^{\infty}(\bar{\Omega})$. However, because of (2.1), it is easy to check that $\Gamma_{v}(x, y)=|x|$, so $\Gamma_{v}$ is not even $C^{1}$.

The best regularity one can expect is $C^{1,1}$. Take $\Omega=[-2,2] \subset \mathbb{R}$ and $v=$ $\left(x^{2}-1\right)^{2}$. Then $\Gamma_{v}(x)=\left[\left(x^{2}-1\right)_{+}\right]^{2}$ is $C^{1,2}$ but not $C^{2}$ inside $\Omega$. 
In Theorem 1.2 the function $v$ needs to be $(1+\alpha)$-semiconcave up to the boundary. Take $\Omega=B(0,1)$ and $v(x, y)$ the harmonic extension of the boundary data $v(\cos \theta, \sin \theta)=|\cos \theta|$. Then by elliptic regularity $v$ is of class $C^{\infty}$ inside $\Omega$, but $\Gamma_{v}(x, y)=|x|$ is not $C^{1}$.

In Theorem 1.2 the boundary regularity of the domain does matter. Take $\Omega=\left\{(x, y):|x|^{p}+|y|^{p} \leq 1\right\}$ for some $p \in(1,2)$, and $v(x, y)=1-y^{2}$. Then $v$ coincides on the boundary of $\Omega$ with $u(x, y)=f(x)=1-\left(1-|x|^{p}\right)^{2 / p}$. Hence, for $\varepsilon>0$ small, the convex function

$$
u_{\varepsilon}(x, y)= \begin{cases}f(-\varepsilon)+f^{\prime}(-\varepsilon)(x+\varepsilon) & \text { if } x \leq-\varepsilon \\ f(x) & \text { if }|x| \leq \varepsilon \\ f(\varepsilon)+f^{\prime}(\varepsilon)(x-\varepsilon) & \text { if } x \geq \varepsilon\end{cases}
$$

is below $v$ in $\Omega$, and thus $\Gamma_{v} \geq u_{\varepsilon}$. Since $\Gamma_{v}(0, y)=0$ and $f \simeq|x|^{p}$ near 0 , this implies that the best regularity we can expect for $\Gamma_{v}$ is $C^{1, p-1}$. (An easy modification of this example gives that in case $\partial \Omega$ is not of class $C^{1}$, then the convex envelope may not be $C^{1}$.)

In Theorem $1.2 v$ has to be globally Lipschitz. Take $\Omega=B(0,1)$, and consider the concave function $v(x, y)=\sqrt{1-|y|^{2}}$. Then $v$ is $(1+\alpha)$-semiconcave for every $\alpha>0$. However, since $v$ is concave and $v=|x|$ on $\partial \Omega$, we get that $\Gamma_{v}(x, y)=|x| \notin C^{1}(\Omega)$.

In Theorem $1.3 C^{3,1}$ regularity of $v$ is necessary. Take $\Omega=B(0,1)$ and $v(x, y)$ the harmonic extension of the $C^{3,1-2 \varepsilon}$ boundary data $v(\cos \theta, \sin \theta)=(1+\cos \theta)^{2-\varepsilon}$. Then by elliptic regularity $v$ is of class $C^{3,1-2 \varepsilon}$ up to the boundary, but $\Gamma_{v}(x, y)=$ $(1+x)^{2-\varepsilon} \notin C^{1,1}(\bar{\Omega})$.

In Theorem 1.3 uniform convexity of $\Omega$ is necessary. Take $\Omega=\{(x, y)$ : $\left.|x|^{4}+|y|^{4} \leq 1\right\}$, and $v$ the harmonic extension of the $C^{7,1-4 \varepsilon}$ boundary data $v(x, y)=(1+x)^{2-\varepsilon}$. Then by elliptic regularity $v$ is of class $C^{7,1-4 \varepsilon}$ up to the boundary, but $\Gamma_{v}(x, y)=(1+x)^{2-\varepsilon} \notin C^{1,1}(\bar{\Omega})$.

In Theorem $1.3 C^{3,1}$ regularity of $\partial \Omega$ is necessary. Let $\Omega$ be a convex with $0 \in \partial \Omega$. We assume that $\partial \Omega \backslash\{0\}$ is of class $C^{\infty}$, that $\Omega \subset\left\{y \geq x^{2}+x^{4-2 \varepsilon}\right\}$, and that close to 0 the boundary of $\Omega$ is represented by the graph of $y=x^{2}+x^{4-2 \varepsilon}$. Then, near the origin we can write

$$
\partial \Omega=\{(x, y): \quad y \geq 0-x(y) \leq x \leq x(y)\}
$$

where $x(y)$ is the inverse of $x^{2}+x^{4-2 \varepsilon}$ for $x \geq 0$. Now consider $v(x, y)=y-x^{2}$. Then

$$
\begin{aligned}
v( \pm x(y), y) & =y-x(y)^{2}=x(y)^{4-2 \varepsilon}=\left[x(y)^{2}\right]^{2-\varepsilon} \\
& =\left[y-x(y)^{4-2 \varepsilon}\right]^{2-\varepsilon}=y^{2-\varepsilon}(1+o(1)) .
\end{aligned}
$$

Hence, since $v>0$ on $\bar{\Omega} \backslash\{0\}$, it is easy to see that

$$
\frac{y^{2-\varepsilon}}{M} \leq v(x, y) \leq M y^{2-\varepsilon} \quad \text { on } \partial \Omega
$$


for some constant $M$ sufficiently large. Moreover, since the function $x \mapsto v(x, y)$ is concave, $\Gamma_{v} \geq y^{2-\varepsilon} / M$ inside $\Omega$. We claim that $\partial_{y} \Gamma_{v}(0,0)=0$. In fact, if this were not the case, for $y>0$ small we would have

$$
\Gamma_{v}(0, y) \geq c y>M y^{2-\varepsilon} \geq \Gamma_{v}( \pm x(y), y),
$$

which is absurd since $\Gamma_{v}$ is convex. It is thus clear that $\Gamma_{v}$ cannot have second derivatives bounded up to the origin, as otherwise we would get

$$
\frac{y^{2-\varepsilon}}{M} \leq \Gamma_{v}(0, y) \leq C y^{2},
$$

which is clearly false for $y$ small.

\section{Notation AND PRELIMINARIES}

From now on, $\Omega$ will denote a bounded open convex set. Given $k \in \mathbb{N}$ and $\alpha \in[0,1]$, we say that $\Omega$ has boundary of class $C^{k, \alpha}$ if there exists a radius $\varrho$ such that for every point $x \in \partial \Omega$, up to a rotation of coordinates, it holds

$$
\Omega \cap B(x, \varrho)=\left\{\left(x^{\prime}, t\right) \in \mathbb{R}^{n-1} \times \mathbb{R}: t \geq \varphi\left(x^{\prime}\right)\right\} \cap B(x, \varrho)
$$

for some convex function $\varphi: \mathbb{R}^{n-1} \rightarrow \mathbb{R}$ of class $C^{k, \alpha}$. Notice that every convex set has boundary at least of class $C^{0,1}$.

When $\Omega$ has boundary of class at least $C^{1}$, we will denote by $\nu_{x}$ its outer normal at a point $x \in \partial \Omega$. Moreover, we say that a $C^{1}$ convex set is uniformly convex if there exists $R>0$ such that

$$
\Omega \subset B\left(x_{0}-R \nu_{x_{0}}, R\right) \quad \forall x_{0} \in \partial \Omega .
$$

Given a set $\mathcal{C}$ we will denote by $\operatorname{conv}(\mathcal{C})$ its closed convex hull. In case $\mathcal{C}=$ $\left\{x_{1}, \ldots, x_{k}\right\}$ is a finite set of points, we will simply write $\operatorname{conv}\left(x_{1}, \ldots, x_{k}\right)$. The segment between two points $x_{1}$ and $x_{2}$ will be indicated by $\left[x_{1}, x_{2}\right]$.

Given a continuous function $v: \bar{\Omega} \rightarrow \mathbb{R}$, we say that a "slope" $s_{x_{0}} \in \mathbb{R}^{n}$ is supporting $v$ at $x_{0} \in \bar{\Omega}$ if

$$
v(x) \geq v\left(x_{0}\right)+s_{x_{0}} \cdot\left(x-x_{0}\right) \quad \text { for every } x \in \bar{\Omega} .
$$

In case $s_{x_{0}}$ supports $v$ at $x_{0}$, we will write $x_{0} \in \operatorname{Dom}(\partial v)$ and $s_{x_{0}} \in \partial v\left(x_{0}\right)$. Notice that the function $v(x)-v\left(x_{0}\right)+s_{x_{0}} \cdot\left(x-x_{0}\right)$ has a minimum at $x_{0}$. We also recall that a convex function can be characterized by $\operatorname{Dom}(\partial v)=\Omega$.

Given a $(1+\alpha)$-semiconcave function (see Definition 1.1), we observe that, for any $x_{0} \in \bar{\Omega}$, the set of slopes $p_{x_{0}}$ which satisfy (1.2) is a convex subset of $\mathbb{R}^{n}$. This allows us to choose an "optimal slope" $\bar{p}_{x_{0}}$ as the element of minimal norm inside such set. The reason for this choice is the following observation, whose elementary proof is left to the reader: if $v$ is Lipschitz continuous, then

$$
\sup _{x_{0} \in \bar{\Omega}}\left|\bar{p}_{x_{0}}\right| \leq \operatorname{Lip} v \text {. }
$$

It is easy to see that if $x_{0} \in \Omega \cap \operatorname{Dom}(\partial v)$ and $v$ is $(1+\alpha)$-semiconcave, then $v$ is differentiable at $x_{0}$ with

$$
\nabla v\left(x_{0}\right)=p_{x_{0}}=s_{x_{0}} .
$$

In case $x_{0} \in \partial \Omega$ and $\partial \Omega$ is at least of class $C^{1}$, we have

$$
p_{x_{0}}=s_{x_{0}}-\lambda \nu_{x_{0}}
$$

where $\nu_{x_{0}}$ is the outer normal at $x_{0}$ and $\lambda \geq 0$. 
Finally, given a closed set $\mathcal{K} \subset \mathbb{R}^{n}$, we say that a function $v: \mathcal{K} \rightarrow \mathbb{R}$ is $C^{k, \alpha}(\mathcal{K})$ if there exists a $C^{k, \alpha}$ extension of $v$ in a neighborhood of $\mathcal{K}$.

We now give two criteria to show $C^{1, \alpha}$ regularity. The following lemmas are pretty standard (see for instance [5] Section 1]), but for the sake of completeness we provide the proofs.

Lemma 3.1. Let $\Omega$ be a convex open set, $C$ and $\bar{\varrho}$ be positive constants, and $\alpha \in(0,1]$. Let $u: \Omega \rightarrow \mathbb{R}$ be a globally Lipschitz convex function such that for every $x \in \Omega$ there exists a supporting slope $s_{x}$ satisfying

$$
u(y)-u(x)-s_{x} \cdot(y-x) \leq C|y-x|^{1+\alpha} \quad \text { for all } y \in \Omega \cap B(x, \bar{\varrho}) .
$$

Then $u \in C^{1, \alpha}(\bar{\Omega})$.

Proof. First of all notice that, by convexity and (3.4), $u$ is differentiable at every point in $\Omega$ with $\nabla u(x)=s_{x}$, and

$$
|u(y)-u(x)-\nabla u(x) \cdot(y-x)| \leq C|y-x|^{1+\alpha} \quad \text { for all } y \in \Omega \cap B(x, \bar{\varrho}) .
$$

We now show that there exists a constant $M>0$ such that

$$
|\nabla u(x)-\nabla u(y)| \leq M|x-y|^{\alpha} \quad \text { for all } x, y \in \Omega .
$$

Since in the above estimate $M$ is independent of $x$ and $y$, this implies that $u$ extends to a $C^{1, \alpha}$ function up to the boundary.

Because by assumption $u$ is Lipschitz, it is enough to show (3.6) for $|x-y|$ small. Now recall that since $\Omega$ is convex, $\partial \Omega$ is Lipschitz. Hence there exist $\varrho_{0} \in(0, \bar{\varrho} / 2)$ and $\eta>0$ such that, for any $x \in \Omega$, for any vector $e$, and for any $\varrho \leq \varrho_{0}$, there is a point $z \in B\left(x, \varrho_{0}\right) \cap \Omega$ satisfying

$$
|e \cdot(z-x)| \geq \eta|z-x||e| \text { and }|z-x|=\varrho .
$$

Up to subtracting a linear function, without loss of generality we can assume that $\nabla u(x)=0$. We want to show that

$$
|\nabla u(y)| \leq M|y-x|^{\alpha} \quad \text { for all } y \in B\left(x, \varrho_{0}\right)
$$

for some constant $M>0$ independent of $x$. Fix a point $z \in B\left(y, \varrho_{0}\right) \cap \Omega$ such that

$$
|\nabla u(y) \cdot(z-y)| \geq \eta|z-y||\nabla u(y)| \text { and }|z-y|=|y-x| .
$$

Thanks to (3.5) we have

$$
\begin{aligned}
\eta|z-y||\nabla u(y)| & \leq|\nabla u(y) \cdot(z-y)| \\
& \leq|u(z)-u(y)-\nabla u(y) \cdot(z-y)| \\
& +|u(z)-u(x)|+|u(y)-u(x)| \\
& \leq C\left(|z-y|^{1+\alpha}+|z-x|^{1+\alpha}+|y-x|^{1+\alpha}\right) .
\end{aligned}
$$

Since $|z-y|=|y-x|$, this gives (3.7) with $M=\left(2+2^{1+\alpha}\right) C$.

In case one wants to prove $C^{1,1}$ regularity, the above lemma can be refined as follows (see [5, Section 1]).

Lemma 3.2. Let $\Omega$ be a convex open set, $C>0$, and $u: \Omega \rightarrow \mathbb{R}$ be a globally Lipschitz convex function such that for every $x \in \Omega$ there exist a supporting slope $s_{x}$ and a radius $\varrho(x)$ satisfying

$$
u(y)-u(x)-s_{x} \cdot(y-x) \leq C|y-x|^{2} \quad \text { for all } y \in \Omega \cap B(x, \varrho(x)) .
$$

Then $u \in C^{1,1}(\bar{\Omega})$. 
Notice that in (3.8) the radius $\varrho(x)$ can depend on the point $x$, while in (3.4) the radius has to be uniform. It is indeed easy to see that the uniform assumption in (3.4) is necessary.

Proof. Thanks to Lemma 3.1 it suffices to show that (3.8) implies that

$$
u(y)-u(x)-\nabla u(x) \cdot(y-x) \leq 2 C|y-x|^{2} \quad \text { for all } x, y \in \Omega .
$$

In fact suppose there exists a point $\bar{y}$ where (3.9) fails, and along the segment $[x, \bar{y}]$ let us consider the following function:

$$
f(t)=2 C|\bar{y}-x|^{2} t^{2}-u(x+t(\bar{y}-x))+u(x)+t \nabla u(x) \cdot(\bar{y}-x) .
$$

Since $f(t)>0$ for $t>0$ small, $f(1)<0$, and $f(0)=0$, there exists a maximum point $\bar{t} \in(0,1)$. Thus for any $h>0$ small we have

$$
\begin{aligned}
0 & \geq f(\bar{t}+h)+f(\bar{t}-h)-2 f(\bar{t}) \\
& =2 C|\bar{y}-x|^{2}\left((\bar{t}+h)^{2}+(\bar{t}-h)^{2}-2 \bar{t}^{2}\right) \\
& -\{u(x+(\bar{t}+h)(\bar{y}-x))+u(x+(\bar{t}-h)(\bar{y}-x))-2 u(x+\bar{t}(\bar{y}-x))\} .
\end{aligned}
$$

Since for $h$ small the term in curly brackets is bounded by $2 C|x-y|^{2} h^{2}$ (by (3.8)), we obtain

$$
h^{2} \geq(\bar{t}+h)^{2}+(\bar{t}-h)^{2}-2 \bar{t}^{2}=2 h^{2},
$$

a contradiction.

We close this section with the following well-known lemma. To make the presentation self-contained we provide its simple proof.

Lemma 3.3. Let $v \in C(\bar{\Omega})$, and fix $x \in \Omega \backslash\left\{v=\Gamma_{v}\right\}$. Then there exist at most $n+1$ points $x_{1}, \ldots, x_{n+1}$ in $\bar{\Omega} \cap\left\{v=\Gamma_{v}\right\}$ such that

$$
x \in \operatorname{conv}\left(x_{1}, \ldots, x_{n+1}\right) .
$$

Moreover every supporting slope to $\Gamma_{v}$ at $x$ is a supporting slope for $v$ at $x_{i}$.

Proof. Let $s_{x}$ be a supporting slope to $\Gamma_{v}$ at $x$ and define

$$
\mathcal{C}=\left\{y \in \bar{\Omega}: v(y)=\Gamma_{v}(x)+s_{x}(y-x)\right\} .
$$

We claim that $x \in \operatorname{conv}(\mathcal{C})$. Suppose it is not true, then there exists an affine function $\ell$ which strongly separates $\mathcal{C}$ and $x$ : more precisely $\ell(x)>0$ and $\ell(y)<0$ for every $y$ in $\mathcal{C}$. It is now easy to see that, for some small $\delta>0$, the function

$$
\tilde{\ell}(y)=\Gamma_{v}(x)+s_{x}(y-x)+\delta \ell(y)
$$

is below $v$ in $\bar{\Omega}$ but $\tilde{\ell}(x)>\Gamma_{v}(x)$, against the definition of $\Gamma_{v}$. The existence of at most $(n+1)$-points follows from the Carathéodory Theorem [15. Theorem 17.1]. Finally it is immediate to check that $s_{x}$ is supporting $v$ at $x_{i}$. 


\section{Proof of Theorem 1.2}

Proof. Fix $\delta_{0}>0$. We want to show that $\Gamma_{v}$ is of class $C^{1, \min \{\alpha, \beta\}}$ inside $\{x \in \Omega$ : $\left.\operatorname{dist}\left(x_{0}, \partial \Omega\right) \geq \delta_{0}\right\}$.

Pick $x_{0} \in \Omega$ with $\operatorname{dist}\left(x_{0}, \partial \Omega\right) \geq \delta_{0}$, and let $s_{x_{0}}$ be a supporting slope to $\Gamma_{v}$ at $x_{0}$. Up to subtracting an affine function we can assume that

$$
\left|s_{x_{0}}\right|=\Gamma_{v}\left(x_{0}\right)=0 .
$$

Then by Lemma 3.1 it suffices to prove that there exist positive constants $M$ and $\varrho_{1}$, depending only on $\delta_{0}$, such that

$$
\Gamma_{v}\left(x_{0}+h\right) \leq M|h|^{1+\min \{\alpha, \beta\}} \quad \text { for all }|h| \leq \varrho_{1} .
$$

In the case $x_{0} \in\left\{v=\Gamma_{v}\right\}$ we have $0=s_{x_{0}}=\nabla v\left(x_{0}\right)$, so by the $(1+\alpha)$ semiconcavity

$$
\Gamma_{v}\left(x_{0}+h\right) \leq v\left(x_{0}\right)+C|h|^{1+\alpha}=C|h|^{1+\alpha}
$$

for $|h| \leq \varrho_{0}$ and we are done.

Now suppose $x_{0} \in \Omega \backslash\left\{v=\Gamma_{v}\right\}$. By Lemma 3.3 there exist $n+1$ points $x_{i} \in\left\{v=\Gamma_{v}\right\} \cap \bar{\Omega}$ such that

$$
x_{0}=\sum_{j=1}^{n+1} \lambda_{j} x_{j}, \quad \lambda_{j} \geq 0, \quad \sum_{j=1}^{n+1} \lambda_{j}=1,
$$

and 0 is a supporting slope at $x_{i}$. Now take $\delta_{1} \in\left(0, \delta_{0} / 4\right)$ small (to be fixed later), and let us divide the set of indexes in two parts: $\{1, \ldots, n+1\}=J_{g} \cup J_{b}$ with

$$
J_{g}=\left\{j: \lambda_{j} \geq \delta_{1}\right\}, \quad J_{b}=\{1, \ldots, n+1\} \backslash J_{g} .
$$

Notice that, since $\sum_{j} \lambda_{j}=1$, the set $J_{g}$ of "good" indexes is non-empty provided we choose $\delta_{1} \leq 1 /(n+1)$. We now distinguish two cases.

Case 1: There exists $\bar{j} \in J_{g}$ such that $\operatorname{dist}\left(x_{\bar{j}}, \partial \Omega\right) \geq \delta_{0} / 4$. Up to reordering, we can assume $\bar{j}=1$. In this case, thanks to (4.1) we have that

$$
\Gamma_{v}\left(x_{1}+h\right) \leq v\left(x_{1}+h\right) \leq C|h|^{1+\alpha} \quad \forall|h| \leq \bar{\varrho}:=\min \left\{\varrho_{0}, \delta_{0} / 4\right\} .
$$

Thus, using the convexity of $\Gamma_{v}$, for $|h| \leq \delta_{1} \bar{\varrho}$ we get

$$
\begin{aligned}
\Gamma_{v}\left(x_{0}+h\right) & =\Gamma_{v}\left(\lambda_{1}\left(x_{1}+\frac{h}{\lambda_{1}}\right)+\sum_{j=2}^{n+1} \lambda_{j} x_{j}\right) \leq \lambda_{1} \Gamma_{v}\left(x_{1}+\frac{h}{\lambda_{1}}\right) \\
& \leq \frac{C}{\lambda_{1}^{\alpha}}|h|^{1+\alpha} \leq \frac{C}{\delta_{1}^{\alpha}}|h|^{1+\alpha},
\end{aligned}
$$

where we have used that $\lambda_{1} \geq \delta_{1}$, and that $\Gamma_{v}\left(x_{i}\right)=0$ for $i=1, \ldots, n+1$.

Case 2: $d\left(x_{j}, \partial \Omega\right) \leq \delta_{0} / 4$ for all $j \in J_{g}$. Let us define

$$
\tilde{x}_{0}=\frac{1}{\sum_{j \in J_{g}} \lambda_{j}} \sum_{j \in J_{g}} \lambda_{j} x_{j}
$$

the "projection" of $x_{0}$ onto the simplex $\Sigma$ generated by the $\left\{x_{j}\right\}_{j \in J_{g}}$ (see Figure 4.1). 


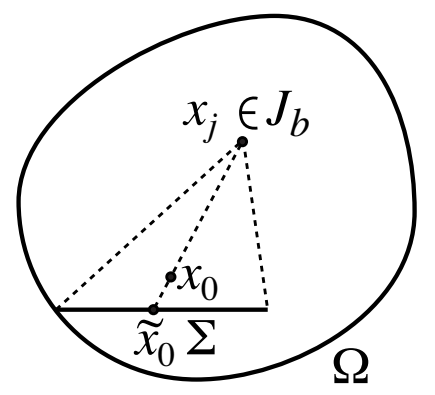

FigurE 4.1. The projection of $x_{0}$ onto the simplex $\Sigma$ generated by $\left\{x_{j}\right\}_{j \in J_{g}}$.

Clearly $\tilde{x}_{0}$ belongs to $\Omega$. Moreover, since for any $y \in \Omega$

$$
\begin{aligned}
\tilde{x}_{0}-x_{0} & =\frac{1}{\sum_{j \in J_{g}} \lambda_{j}} \sum_{j \in J_{g}} \lambda_{j} x_{j}-\sum_{j \in J_{g}} \lambda_{j} x_{j}-\sum_{j \in J_{b}} \lambda_{j} x_{j} \\
& =\frac{1}{\sum_{j \in J_{g}} \lambda_{j}} \sum_{j \in J_{g}} \lambda_{j}\left(x_{j}-y\right)-\sum_{j \in J_{g}} \lambda_{j}\left(x_{j}-y\right)-\sum_{j \in J_{b}} \lambda_{j}\left(x_{j}-y\right) \\
& =\left(\frac{1}{\sum_{j \in J_{g}} \lambda_{j}}-1\right) \sum_{j \in J_{g}} \lambda_{j}\left(x_{j}-y\right)-\sum_{j \in J_{b}} \lambda_{j}\left(x_{j}-y\right),
\end{aligned}
$$

we have

$$
\begin{aligned}
\left|x_{0}-\tilde{x}_{0}\right| & \leq \frac{\sum_{j \in J_{b}} \lambda_{j}}{\sum_{j \in J_{g}} \lambda_{j}} \sum_{j \in J_{g}} \lambda_{j} \operatorname{diam}(\Omega)+\sum_{j \in J_{b}} \lambda_{j} \operatorname{diam}(\Omega) \\
& =2 \operatorname{diam}(\Omega) \sum_{j \in J_{b}} \lambda_{j} \leq 2(n+1) \operatorname{diam}(\Omega) \delta_{1} \leq \frac{\delta_{0}}{4},
\end{aligned}
$$

provided $\delta_{1}$ is small enough. Recalling that $\operatorname{dist}\left(x_{0}, \partial \Omega\right) \geq \delta_{0}$, this implies in particular $\operatorname{dist}\left(\tilde{x}_{0}, \partial \Omega\right) \geq 3 \delta_{0} / 4$.

The strategy is now the following: we first show that

$$
\Gamma_{v}\left(\tilde{x}_{0}+h\right) \leq M|h|^{1+\min \{\alpha, \beta\}} \quad \forall|h| \leq \varrho_{1},
$$

for some constants $M$ and $\bar{\varrho}_{1}$ depending only on $\delta_{0}$, and then we "propagate" this estimate to $x_{0}$.

To prove (4.3), we need to distinguish between two further cases.

Case 2-a: There exists $\bar{j} \in J_{g}$ such that $x_{\bar{j}} \in \Omega$. Up to reordering, we can assume $\bar{j}=1$. Hence $x_{1} \in \Omega$, which implies in particular that $\nabla v\left(x_{1}\right)=0$. Consider $\bar{x}$, the point of intersection of the boundary of $\Sigma$ with the line through $\tilde{x}_{0}$ and $x_{1}$ (see Figure 4.2). More precisely, defining

$$
\mu_{j}=\frac{\lambda_{j}}{\sum_{j \in J_{g}} \lambda_{j}} \quad \forall j \in J_{g},
$$

we have

$$
\bar{x}=\frac{1}{1-\mu_{1}} \sum_{j \in J_{g} \backslash\{1\}} \mu_{j} x_{j}
$$




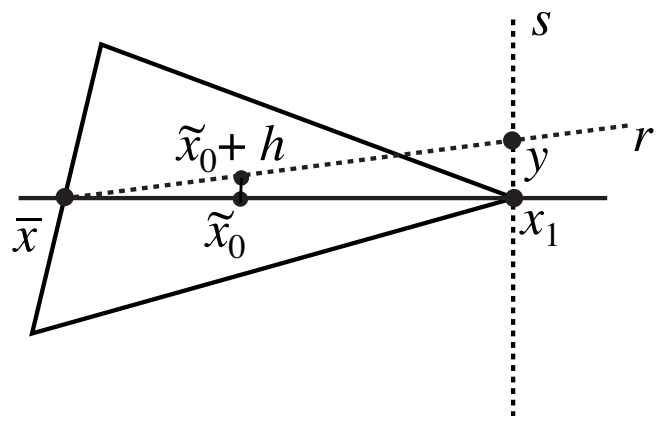

Figure 4.2. To estimate the value of $\Gamma_{v}$ at $\tilde{x}_{0}+h$, we use the value of $v$ at $y$ together with the convexity of $\Gamma_{v}$.

so that $\tilde{x}_{0}=\left(1-\mu_{1}\right) \bar{x}+\mu_{1} x_{1}$. Notice that

$$
\mu_{1}=\frac{\lambda_{1}}{\sum_{j \in J_{g}} \lambda_{j}} \geq \frac{\delta_{1}}{1-(n+1) \delta_{1}},
$$

and $\Gamma_{v}(\bar{x})=0$.

Now choose $|h| \leq \bar{\varrho}$, where $\bar{\varrho}$ is small enough (to be fixed later). If $h$ is parallel to the segment $\left[\bar{x}, x_{1}\right]$ we trivially have

$$
\Gamma_{v}\left(\tilde{x}_{0}+h\right)=0 \quad \text { provided } \bar{\varrho}<\min \left\{\left|\bar{x}-\tilde{x}_{0}\right|,\left|x_{1}-\tilde{x}_{0}\right|\right\} .
$$

Notice that $\min \left\{\left|\bar{x}-\tilde{x}_{0}\right|,\left|x_{1}-\tilde{x}_{0}\right|\right\}$ is bounded from below by a constant depending only on $\delta_{0}$, since so are $\left|x_{1}-\tilde{x}_{0}\right|$ and $\mu_{1}$.

In case $h$ is orthogonal to the segment $\left[\bar{x}, x_{1}\right]$, let us draw the half-line $r$ from $\bar{x}$ through $\tilde{x}_{0}+h$. Let us also consider the line $s$ through $x_{1}$ parallel to $h$. Two things can happen: $r$ meets $s$ inside $\Omega$ or not.

In the first case call $y$ the point of intersection (see Figure 4.2). By similarity and (4.4)

$$
\left|x_{1}-y\right| \leq K|h|, \quad \text { where } \quad K=\frac{\left|\bar{x}-x_{1}\right|}{\left|\bar{x}-\tilde{x}_{0}\right|}=\frac{1}{\mu_{1}} \leq \frac{2}{\delta_{1}}
$$

and

$$
\tilde{x}_{0}+h=\mu_{1} y+\left(1-\mu_{1}\right) \bar{x} .
$$

Hence

$$
\begin{aligned}
\Gamma_{v}\left(\tilde{x}_{0}+h\right) & =\Gamma_{v}\left(\mu_{1} y+\left(1-\mu_{1}\right) \bar{x}\right) \\
& \leq \mu_{1} \Gamma_{v}\left(x_{1}+\left(y-x_{1}\right)\right) \leq v\left(x_{1}+\left(y-x_{1}\right)\right) \leq M|h|^{1+\alpha},
\end{aligned}
$$

where we have used that $\Gamma_{v}(\bar{x})=0$.

In the second case let $z$ be the intersection between $r$ and $\partial \Omega$ (see Figure 4.3). The angle $\sigma$ between $\left[x_{1}, z\right]$ and $\left[\bar{x}, x_{1}\right]$ is bounded from above by $\pi / 2$ and from below by a universal constant. In fact, by convexity of $\Omega$, the convex envelope of $\left\{x_{1}\right\} \cup B\left(\tilde{x}_{0}, \delta_{0} / 2\right)$ is contained in $\Omega$, thus

$$
\sigma \geq \arctan \left(\frac{\delta_{0}}{2 \operatorname{diam}(\Omega)}\right) .
$$




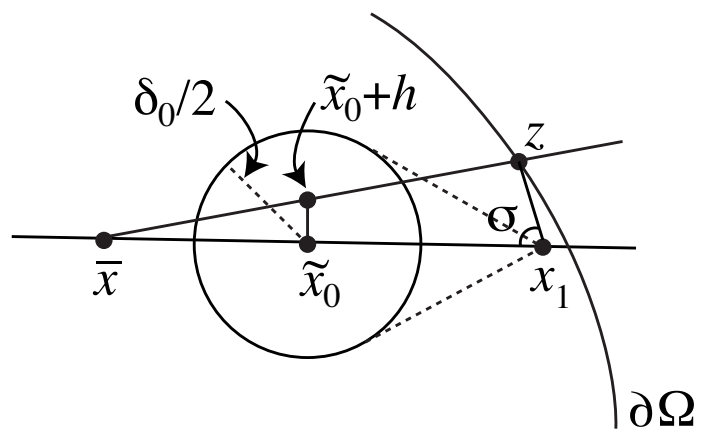

FIGURE 4.3. Since the angle $\sigma$ is uniformly bounded away from 0 , $\left|z-x_{1}\right| \leq K^{\prime}|h|$ for some constant $K^{\prime}>0$ depending only on $\delta_{0}$ and $\operatorname{diam}(\Omega)$, and we conclude as in the previous case.

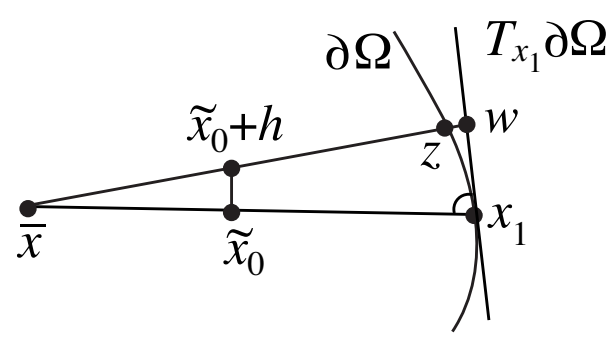

FiguRE 4.4. When $x_{1} \in \partial \Omega$, it is crucial to also exploit the regularity of $\partial \Omega$.

Hence $\left|x_{1}-z\right| \leq K^{\prime}|h|$ for some universal constant $K^{\prime}>0$. Since $\tilde{x}_{0}+h=$ $\gamma y+(1-\gamma) \bar{x}$ for some $\gamma \in(0,1)$, exactly as above we get

$$
\Gamma_{v}\left(\tilde{x}_{0}+h\right) \leq M|h|^{1+\alpha} .
$$

This proves an estimate from above on $\Gamma_{v}\left(\tilde{x}_{0}+h\right)$ whenever $|h| \leq \bar{\varrho}$ is either orthogonal or parallel to $\left[\bar{x}, x_{1}\right]$. Finally, for any $|h| \leq \bar{\varrho} / 2$ write it as $h=h^{p}+h^{o}=$ $\frac{2 h^{p}+2 h^{o}}{2}$, with $h^{p}$ perpendicular to $\left[x_{1}, x_{2}\right]$ and $h^{o}$ parallel to $\left[\bar{x}, x_{1}\right]$. Then, by convexity of $\Gamma_{v}$ we obtain

$$
\begin{aligned}
\Gamma_{v}\left(\tilde{x}_{0}+h\right) & \leq \frac{1}{2}\left(\Gamma_{v}\left(\tilde{x}_{0}+2 h^{p}\right)+\Gamma_{v}\left(\tilde{x}_{0}+2 h^{p}\right)\right) \\
& \leq 2 M\left(\left|h^{p}\right|^{1+\alpha}+\left|h^{o}\right|^{1+\alpha}\right) \leq 4 M|h|^{1+\alpha} .
\end{aligned}
$$

Case 2-b: $x_{j} \in \partial \Omega$ for all $j \in J_{g}$. Pick any of these points, say (up to reordering) $x_{1}$, and construct the point $\bar{x}$ as in Case 2 -a. We again want to estimate $\Gamma_{v}\left(\tilde{x}_{0}+h\right)$ for $h$ orthogonal to $\left[\bar{x}, x_{1}\right]$ and small enough. As before we draw the half-line from $\bar{x}$ through $\tilde{x}_{0}+h$. This half-line will intersect the boundary of $\Omega$ in a point $z$, and the tangent plane $T_{x_{1}} \partial \Omega$ in a point $w$ (see Figure 4.4). Arguing as above we deduce that the angle between $T_{x_{1}} \partial \Omega$ and the segment through $\left[\bar{x}, x_{1}\right]$ is at least $\sigma_{0}$, where $\sigma_{0}>0$ depends only on $\delta_{0}$ and $\operatorname{diam}(\Omega)$ (see Figure 4.3), so $\left|z-x_{1}\right|,\left|w-x_{1}\right| \leq K|h|$ for some universal constant $K$. In this case we cannot however say that $\nabla v\left(x_{1}\right)=0$ 
(as we did in Case 2-a), and thus the above calculations give only

$$
\Gamma_{v}\left(\tilde{x}_{0}+h\right) \leq \bar{p}_{x_{1}} \cdot\left(z-x_{1}\right)+M|h|^{1+\alpha} .
$$

However, since $v$ is Lipschitz and $s_{x_{1}}=0$ (recall (4.1)) we have

$$
\bar{p}_{x_{1}}=-\lambda \nu_{x_{1}},
$$

where $\nu_{x_{1}}$ is the outer normal to $\Omega$ at $x_{1}$, and $0 \leq \lambda \leq \operatorname{Lip} v$ (see (3.2) and (3.3)). We will thus prove (4.3) if we can show that

$$
\left|\nu_{x_{1}} \cdot\left(z-x_{1}\right)\right| \leq C|h|^{1+\beta} .
$$

This follows from the $C^{1, \beta}$ regularity of $\partial \Omega$. In fact, denoting with $\pi(z)$ the orthogonal projection of $z$ on $T_{x_{1}} \partial \Omega$, we have

$$
\left|\nu_{x_{1}} \cdot\left(z-x_{1}\right)\right| \leq C\left|\pi(z)-x_{1}\right|^{1+\beta} \leq C\left|w-x_{1}\right|^{1+\beta} \leq C K^{1+\beta}|h|^{1+\beta} .
$$

This finally concludes the proof of (4.3).

We now want to prove (4.3) with $\tilde{x}_{0}$ replaced by $x_{0}$. To this aim, let us define

$$
\hat{x}_{0}=\frac{1}{\sum_{j \in J_{b}} \lambda_{j}} \sum_{j \in J_{b}} \lambda_{j} x_{j}
$$

so that

$$
x_{0}=(1-\mu) \hat{x}_{0}+\mu \tilde{x}_{0}, \quad \text { where } \quad \mu=\sum_{j \in J_{g}} \lambda_{j} \geq \delta_{1} .
$$

Then, by convexity of $\Gamma_{v}$ and the fact that $\Gamma_{v}\left(\hat{x}_{0}\right)=0$, we infer that

$$
\Gamma_{v}\left(x_{0}+h\right) \leq \mu \Gamma_{v}\left(\tilde{x}_{0}+\frac{h}{\mu}\right) \leq \frac{M}{\delta_{1}}|h|^{1+\min \{\alpha, \beta\}} \quad \forall|h| \leq \delta_{1} \bar{\varrho}_{1},
$$

concluding the proof.

The above theorem can be extended to general moduli of continuity. More precisely, its very same proof shows that if $v$ is $\omega_{1}$-semiconcave (i.e. (1.2) holds with $C\left|x-x_{0}\right| \omega_{1}\left(\left|x-x_{0}\right|\right)$ in place of $\left.C\left|x-x_{0}\right|^{1+\alpha}\right)$ and $\partial \Omega$ is $C^{1}$ with a modulus of continuity $\omega_{2}$, then for any $\Omega^{\prime} \subset \subset \Omega$ there exists $M=M\left(\Omega^{\prime}, \Omega\right)>0$ such that $\nabla u$ has modulus of continuity $t \mapsto M \max \left\{\omega_{1}(M t), \omega_{2}(M t)\right\}$ inside $\Omega^{\prime}$.

Furthermore, it is clear from the proof above (and from the counterexamples in Section (2) that the main difficulty when proving the regularity of $\Gamma_{v}$ arises when the points $\left\{x_{j}\right\}_{j \in J_{q}}$ (see (4.2) ) are close to the boundary (that is, Case 2 in the proof of Theorem 1.2). However, if for some special reason one is able to exclude that case, then the assumptions of Theorem 1.2 can be weakened, and in particular one does not need to assume any regularity on $\partial \Omega$. For example, one can prove the following classical result:

Theorem 4.1. Let $\Omega$ be a convex bounded domain, and $v: \Omega \rightarrow \mathbb{R}$ be a continuous, locally $(1+\alpha)$-semiconcave function. Assume that $v=0$ on $\partial \Omega$, and that $\inf _{\Omega} v<0$. Then $\Gamma_{v} \in C_{\mathrm{loc}}^{1, \alpha}(\Omega)$.

Proof. Let $K_{m}$ define the increasing family of compact convex sets

$$
K_{m}=\operatorname{conv}(\{x \in \Omega: v(x) \leq-1 / m\}), \quad m \in \mathbb{N} .
$$

We observe that $K_{m}$ is compactly supported inside $\Omega$, and that $\bigcup_{m \in \mathbb{N}} K_{m}=\Omega$. 
Fix $m \in \mathbb{N}$, and assume that $x_{0} \in K_{m} \backslash\left\{\Gamma_{v}=v\right\}$. Using the same notation as in the proof of Theorem 1.2, we claim the following:

There exists $\delta_{1}>0$ small, depending on $n, m$, and $\inf _{\Omega} v$ only, such that $x_{j} \in$ $K_{2 m}$ for at least one $j \in J_{g}$ (see (4.2) ).

Once this claim is proved, the $C^{1, \alpha}$ regularity of $\Gamma_{v}$ inside $K_{m}$ is obtained as in Case 1 in the proof of Theorem 1.2 .

To prove the claim, we first observe that, by convexity of $\Gamma_{v}$, we have

$$
\Gamma_{v} \leq-\frac{1}{m} \quad \text { inside } K_{m}
$$

which implies in particular

$$
v \leq-\frac{1}{m} \quad \text { inside } K_{m} \cap\left\{\Gamma_{v}=v\right\} .
$$

Now let $s_{x_{0}}$ be a supporting slope at $x_{0}$. We first claim that there exists at least one $j \in\{1, \ldots, n+1\}$ such that $x_{j} \in K_{2 m}$. In fact, if this were not the case, since $s_{x_{0}}$ is supporting at $x_{i}$ and $\sum_{j} \lambda_{j}=1$, by (4.6) we get

$$
-\frac{1}{m} \geq \Gamma_{v}\left(x_{0}\right)=\sum_{j=1}^{n+1} \lambda_{j}\left(\Gamma_{v}\left(x_{j}\right)+s_{x_{0}} \cdot\left(x_{0}-x_{j}\right)\right)=\sum_{j=1}^{n+1} \lambda_{j} v\left(x_{j}\right) \geq-\frac{1}{2 m},
$$

a contradiction. Now suppose (again by contradiction) that for all $j$ 's such that $x_{j} \in K_{2 m}$, the corresponding $\lambda_{j}$ satisfies $\lambda_{j} \leq \delta_{1}$. Then, by (4.7) and the same calculation as above,

$$
\begin{aligned}
-\frac{1}{m} & \geq \sum_{j=1}^{n+1} \lambda_{j} v\left(x_{j}\right)=\sum_{x_{j} \in K_{2 m}} \lambda_{j} v\left(x_{j}\right)+\sum_{x_{j} \notin K_{2 m}} \lambda_{j} v\left(x_{j}\right) \\
& \geq-(n+1) \delta_{1} \sup _{\bar{\Omega}}(-v)-\frac{1}{2 m},
\end{aligned}
$$

which is impossible for $\delta_{1}$ sufficiently small. This proves the claim and concludes the proof.

\section{Proof of Theorem 1.3}

Proof. Thanks to Lemma 3.2 (notice that in this case the convex envelope is globally Lipschitz in $\Omega$ ) and arguing as in Case 1 of the proof of Theorem 1.2 it is enough to show that for every $x_{0}$ in $\Omega \backslash\left\{v=\Gamma_{v}\right\}$ there exists a radius $\varrho\left(x_{0}\right)$ such that

$$
\Gamma_{v}(x) \leq \Gamma_{v}\left(x_{0}\right)+s_{x_{0}}\left(x-x_{0}\right)+C\left|x-x_{0}\right|^{2} \quad \forall x \in B\left(x_{0}, \varrho\left(x_{0}\right)\right) \cap \bar{\Omega}
$$

for some universal constant $C$ independent of $x_{0}$. Without loss of generality we can assume

$$
\Gamma_{v}\left(x_{0}\right)=\left|s_{x_{0}}\right|=0 .
$$

Then, using Lemma 3.3 we write $x_{0}$ as a convex combination of $k$ points in $\left\{v=\Gamma_{v}\right\}$, with $k \leq n+1$.

We first show that we can reduce to the case $k=2$. Indeed, suppose that (5.1) holds with a constant $C_{k}$ for every point $x_{0}$ which is a convex combination of at most $k$ points in $\left\{v=\Gamma_{v}\right\}$. We want to show it holds for all points which are a convex combination of $k+1$ points with constant $C_{k+1}=(k+1) C_{k}$. To do this, assume that

$$
x_{0} \in \operatorname{conv}\left(x_{1}, \ldots, x_{k+1}\right)
$$


and write it as

$$
x_{0}=(1-\lambda) x_{1}+\lambda \tilde{x}_{0}
$$

where $\lambda \geq 1 /(k+1)$ and

$$
\tilde{x}_{0} \in \operatorname{conv}\left(x_{1}, \ldots, x_{k}\right) .
$$

(Notice that, up to a relabeling, this is always possible.) Since $k \geq 2$, by uniform convexity of $\Omega$ both $x_{0}$ and $\tilde{x}_{0}$ are interior points. Hence there exists a radius $\varrho\left(x_{0}\right)$ such that $\lambda \varrho\left(x_{0}\right) \leq \varrho\left(\tilde{x}_{0}\right)$, and both balls $B\left(x_{0}, \varrho\left(x_{0}\right)\right)$ and $B\left(\tilde{x}_{0}, \varrho\left(\tilde{x}_{0}\right)\right)$ are contained in $\Omega$. Recalling (5.2) we see that 0 supports $\Gamma_{v}$ both at $x_{1}$ and $\tilde{x}_{0}$. Thus $\Gamma_{v}\left(x_{1}\right)=\Gamma_{v}\left(\tilde{x}_{0}\right)=0$ and we get

$\Gamma_{v}\left(x_{0}+\left(x-x_{0}\right)\right) \leq \lambda \Gamma_{v}\left(\tilde{x}_{0}+\frac{\left(x-x_{0}\right)}{\lambda}\right) \leq(k+1) C_{k}\left|x-x_{0}\right|^{2} \quad$ for $x \in B\left(x_{0}, r\left(x_{0}\right)\right)$.

This proves the validity of (5.1) when $x_{0}$ is the convex combination of at most $k+1$ points.

We are thus left to show (5.1) when $x_{0}$ is the convex combination of only two contact points:

$$
x_{0}=(1-\lambda) x_{1}+\lambda x_{2} .
$$

By symmetry we can assume that $\lambda \geq 1 / 2$. In case $x_{2}$ is an interior point we can argue as above (with $x_{2}$ in place of $\tilde{x}_{0}$ ) to obtain (5.1), so we can assume that $x_{2} \in \partial \Omega$. Up to a change of coordinates we assume that the inner normal to $\partial \Omega$ at $x_{2}$ is given by $e_{n}$ (see Figure 5.1). Let $\theta$ denote the angle between the segment $\left[x_{1}, x_{2}\right]$ and the tangent plane to $\partial \Omega$ at $x_{2}$. By the uniform convexity of $\Omega$ we have that

$$
\left|x_{1}-x_{2}\right| \leq 2 R \theta,
$$

where $R>0$ is such that $\Omega \subset B\left(R e_{n}, R\right)$. Indeed if we draw the half-line from $x_{2}$ to $x_{1}$, this meets the boundary of $B\left(R e_{n}, R\right)$ in a point $x_{3}$ which satisfies $\left|x_{3}-x_{2}\right|=$ $2 R \sin (\theta)$, thus

$$
\left|x_{1}-x_{2}\right| \leq\left|x_{3}-x_{2}\right| \leq 2 R \theta \text {. }
$$

We now distinguish two cases, depending on whether $\theta$ is small or not.

Case 1: $\theta \leq \theta_{0}$, with $\theta_{0}$ universally small. Up to a rotation of coordinate we can also assume that the segment $\left[x_{1}, x_{2}\right]$ lies in the plane generated by $e_{1}, e_{n}$ (denoted by $\left.\Pi_{e_{1}, e_{n}}\right)$.

Let us consider a ball of radius $\varrho\left(x_{0}\right) \ll \theta$ around $x_{0}$, and consider $x \in B\left(x_{0}, \varrho\left(x_{0}\right)\right)$ such that $x-x_{0}$ is orthogonal to $\left[x_{1}, x_{2}\right]$.

In case $x-x_{0}$ is orthogonal to $\Pi_{e_{1}, e_{n}}$, let us draw the half-line from $x_{1}$ through $x$, and let $y$ be its point of intersection with $\partial \Omega$. Then by similarity we get $\left|y-x_{2}\right| \leq$ $2\left|x-x_{0}\right|$ (recall that $\lambda \geq 1 / 2$ ), so by the very same arguments as in Case 2-b of Theorem 1.2 we get

$$
\Gamma_{v}(x) \leq M\left|x-x_{0}\right|^{2} .
$$

On the other hand, if $x-x_{0}$ belongs to the plane $\Pi_{e_{1}, e_{n}}$, then we need to do a more refined argument as outlined in Figure 5.1. First of all, we choose $\theta_{0}$ so small that, if we call $\delta$ the distance between $x_{2}$ and the projection of $x_{1}$ on the tangent plane to $\partial \Omega$ at $x_{2}$, then

$$
\partial \Omega \cap B\left(x_{2}, 2 \delta\right)=\left\{\left(x^{\prime}, \varphi\left(x^{\prime}\right)\right):\left|x^{\prime}-x_{2}^{\prime}\right| \leq 2 \delta\right\},
$$




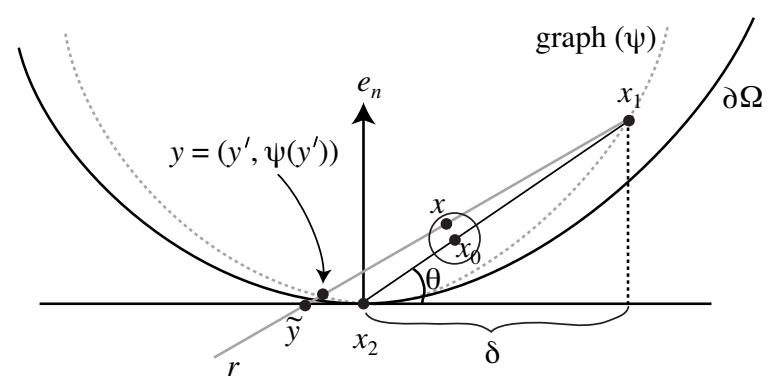

Figure 5.1. To estimate the value of $\Gamma_{v}$ at $x$, we need to show that the function $v$ restricted to the graph of $\psi$ has second derivatives bounded by $C \theta^{2}$ at the point $x_{2}$.

where, by assumption, $\varphi \in C^{3,1}$. We now consider the function

$$
\psi\left(x^{\prime}\right)=\varphi\left(x^{\prime}\right)+\frac{c \tan (\theta)}{\delta}\left|x^{\prime}-x_{2}^{\prime}\right|^{2},
$$

where $c \leq 1$ is chosen such that $x_{1}$ belongs to the graph (obviously $x_{2}$ belongs to it), and define the function $\tilde{v}\left(x^{\prime}\right)=v\left(x^{\prime}, \psi\left(x^{\prime}\right)\right)$. Since $v$ has a minimum at $x_{1}=\left(x_{1}^{\prime}, \psi\left(x_{1}^{\prime}\right)\right)$ and $x_{2}=\left(x_{2}^{\prime}, \psi\left(x_{2}^{\prime}\right)\right)$, $\tilde{v}$ has a minimum at $x_{1}^{\prime}$ and $x_{2}^{\prime}$. This means that $\partial_{1} \tilde{v}=0$ and $\partial_{11} \tilde{v} \geq 0$ at these points. Then, by Rolle's Theorem, there exists a point $x_{3}^{\prime} \in\left[x_{2}^{\prime}, x_{1}^{\prime}\right]$ where $\partial_{11} \tilde{v}\left(x_{3}^{\prime}\right)=0$, and since $\partial_{11} \tilde{v}$ is non-negative at the extremes of the segment, it must have an interior minimum. This implies that there exists a point in $\left[x_{2}^{\prime}, x_{1}^{\prime}\right]$ where $\partial_{111} \tilde{v}$ vanishes. Thus, integrating twice along the segment we obtain

$$
\sup _{\left[x_{2}^{\prime}, x_{1}^{\prime}\right]}\left|\partial_{11} \tilde{v}\right| \leq \delta^{2} \sup _{\left[x_{2}^{\prime}, x_{1}^{\prime}\right]}\left|\partial_{1111} \tilde{v}\right|
$$

We now want to estimate the supremum of the fourth derivatives: a tedious but straightforward calculation gives

$$
\left|\partial_{1111} \tilde{v}\right| \leq C\left(1+\left|\partial_{1} \psi\right|^{4}+\left|\partial_{1} \psi\right|^{2}\left|\partial_{11} \psi\right|+\left|\partial_{11} \psi\right|^{2}+\left|\partial_{1} \psi\right|\left|\partial_{111} \psi\right|+\left|\partial_{1111} \psi\right|\right),
$$

for some universal constant $C$. Notice that, due to the particular form of $\psi$, the above expression is bounded by $C^{\prime}\left(1+(\theta / \delta)^{2}\right)$, where $C^{\prime}$ is also universal. Combining all together we infer that

$$
\sup _{\left[x_{2}^{\prime}, x_{1}^{\prime}\right]}\left|\partial_{11} \tilde{v}\right| \leq C^{\prime}\left(\delta^{2}+\theta^{2}\right)
$$

which implies in particular

$$
\left|\partial_{11} \tilde{v}\left(x_{2}^{\prime}\right)\right| \leq C^{\prime}\left(\delta^{2}+\theta^{2}\right) \leq C^{\prime \prime} \theta^{2}
$$

where in the last inequality we used that $\delta \leq 2 R \theta$ (see (5.3)) ).

Now consider the point $y$ obtained by intersecting the half-line $r$ from $x_{1}$ through $x$ with the graph of $\psi$, and let $y^{\prime}$ denote its projection onto the tangent plane at $x_{2}$ (see Figure 5.1). Clearly $x$ is a convex combination of $x_{1}$ and $y$, thus (since 


$$
\begin{aligned}
\Gamma_{v}\left(x_{1}\right)=0 \text { and } \partial_{1} \tilde{v}\left(x_{2}^{\prime}\right)= & 0) \\
\Gamma_{v}(x) & \leq \Gamma_{v}(y) \leq v\left(y^{\prime}, \psi\left(y^{\prime}\right)\right)=\tilde{v}\left(y^{\prime}\right) \\
& \leq \frac{1}{2} \partial_{11} \tilde{v}\left(x_{2}^{\prime}\right)\left|y^{\prime}-x_{2}^{\prime}\right|^{2}+C\left|y^{\prime}-x_{2}^{\prime}\right|^{3} \\
& \leq K\left(\theta^{2}\left|y^{\prime}-x_{2}^{\prime}\right|^{2}+\left|y^{\prime}-x_{2}^{\prime}\right|^{3}\right),
\end{aligned}
$$

for some universal constant $K>0$. We now observe that, if we call $\tilde{y}$ the intersection of $r$ with the tangent plane at $x_{2}$, then by choosing $\varrho\left(x_{0}\right)$ sufficiently small we get

$$
\left|y^{\prime}-x_{2}^{\prime}\right| \leq 2\left|\tilde{y}-x_{2}\right| \leq \frac{4\left|x-x_{0}\right|}{\sin (\theta / 2)} \leq \frac{10\left|x-x_{0}\right|}{\theta},
$$

where in the last inequality we used that $\theta$ is small. Hence,

$$
\Gamma_{v}(x) \leq K^{\prime}\left|x-x_{0}\right|^{2}+K^{\prime} \frac{\left|x-x_{0}^{\prime}\right|^{3}}{\theta^{3}} \quad \forall x \in B\left(x_{0}, \varrho\left(x_{0}\right)\right),
$$

and the desired estimate follows by choosing $\varrho\left(x_{0}\right) \leq \theta^{3}$.

This proves that

$$
\Gamma_{v}(x) \leq M\left|x-x_{0}\right|^{2}
$$

whenever $x-x_{0} \in B\left(0, \varrho\left(x_{0}\right)\right)$ is orthogonal to $\left[x_{1}, x_{2}\right]$, and either it belongs to $\Pi_{e_{1}, e_{n}}$ or it is orthogonal to it. A simple argument based on the convexity of $\Gamma_{v}$ (as the one used at the end of Case 2-a in the proof of Theorem 1.2) completes the proof.

Case 2: $\theta \geq \theta_{0}$. This case is much simpler: indeed, if $x \in B\left(x_{0}, \varrho\left(x_{0}\right)\right)$ and we define $y$ to be the intersection of the half-line from $x_{1}$ through $x$ with $\partial \Omega$, then by choosing $\varrho\left(x_{0}\right)$ sufficiently small we get

$$
\left|y-x_{2}\right| \leq \frac{10\left|x-x_{0}\right|}{\theta_{0}} .
$$

Hence, by convexity of $\Gamma_{v}$ and the fact that $\Gamma_{v}\left(x_{1}\right)=0$, we obtain

$$
\Gamma_{v}(x) \leq \Gamma_{v}(y) \leq C\left|y-x_{2}\right|^{2} \leq \frac{100 C\left|x-x_{0}\right|^{2}}{\theta_{0}^{2}} \quad \forall x \in B\left(x_{0}, \varrho\left(x_{0}\right)\right),
$$

which concludes the proof.

\section{ACKNOWLEDGMENTS}

The authors thank Maria Colombo and Berardo Ruffini for a careful reading of a preliminary version of this paper and for several useful comments. The second author has been partially supported by NSF Grant DMS-0969962. Both authors acknowledge the support of the ERC ADG Grant GeMeThNES.

\section{REFERENCES}

[1] J. Benoist and J.-B. Hiriart-Urruty, What is the subdifferential of the closed convex hull of a function?, SIAM J. Math. Anal. 27 (1996), no. 6, 1661-1679, DOI 10.1137/S0036141094265936. MR1416513 (97k:49026)

[2] Patrick Bernard, Lasry-Lions regularization and a lemma of Ilmanen, Rend. Semin. Mat. Univ. Padova 124 (2010), 221-229, DOI 10.4171/RSMUP/124-15. MR2752687|(2012d:37191)

[3] Luis A. Caffarelli, Interior $W^{2, p}$ estimates for solutions of the Monge-Ampère equation, Ann. of Math. (2) 131 (1990), no. 1, 135-150, DOI 10.2307/1971510. MR1038360 (91f:35059)

[4] Luis A. Caffarelli and Xavier Cabré, Fully nonlinear elliptic equations, American Mathematical Society Colloquium Publications, vol. 43, American Mathematical Society, Providence, RI, 1995. MR:1351007(96h:35046) 
[5] L. Caffarelli, L. Nirenberg, and J. Spruck, The Dirichlet problem for the degenerate MongeAmpère equation, Rev. Mat. Iberoamericana 2 (1986), no. 1-2, 19-27, DOI 10.4171/RMI/23. MR864651 (87m:35103)

[6] Pierre Cardaliaguet, Front propagation problems with nonlocal terms. II, J. Math. Anal. Appl. 260 (2001), no. 2, 572-601, DOI 10.1006/jmaa.2001.7483. MR.1845570 (2002f:35116)

[7] Guido De Philippis and Alessio Figalli, Second order stability for the Monge-Ampère equation and strong Sobolev convergence of optimal transport maps, Anal. PDE 6 (2013), no. 4, 9931000, DOI 10.2140/apde.2013.6.993. MR3092736

[8] A. Fathi and M. Zavidovique, Ilmanen's lemma on insertion of $C^{1,1}$ functions, Rend. Semin. Mat. Univ. Padova 124 (2010), 203-219, DOI 10.4171/RSMUP/124-14. MR 2752686 (2012i:58004)

[9] David Gilbarg and Neil S. Trudinger, Elliptic partial differential equations of second order, Classics in Mathematics, Springer-Verlag, Berlin, 2001. Reprint of the 1998 edition. MR.1814364 (2001k:35004)

[10] A. Griewank and P. J. Rabier, On the smoothness of convex envelopes, Trans. Amer. Math. Soc. 322 (1990), no. 2, 691-709, DOI 10.2307/2001721. MR986024(91k:49021)

[11] Bernd Kirchheim and Jan Kristensen, Differentiability of convex envelopes (English, with English and French summaries), C. R. Acad. Sci. Paris Sér. I Math. 333 (2001), no. 8, 725728, DOI 10.1016/S0764-4442(01)02117-6. MR1868942 (2002g:49024)

[12] Tom Ilmanen, The level-set flow on a manifold, Differential geometry: partial differential equations on manifolds (Los Angeles, CA, 1990), Proc. Sympos. Pure Math., vol. 54, Amer. Math. Soc., Providence, RI, 1993, pp. 193-204. MR1216585 (94d:58040)

[13] Adam M. Oberman, The convex envelope is the solution of a nonlinear obstacle problem, Proc. Amer. Math. Soc. 135 (2007), no. 6, 1689-1694 (electronic), DOI 10.1090/S0002-993907-08887-9. MR2286077 (2007k:35184)

[14] Adam M. Oberman and Luis Silvestre, The Dirichlet problem for the convex envelope, Trans. Amer. Math. Soc. 363 (2011), no. 11, 5871-5886, DOI 10.1090/S0002-9947-2011-05240-2. MR2817413 (2012e:35064)

[15] R. Tyrrell Rockafellar, Convex analysis, Princeton Landmarks in Mathematics, Princeton University Press, Princeton, NJ, 1997. Reprint of the 1970 original; Princeton Paperbacks. MR1451876 (97m:49001)

Scuola Normale Superiore, P.za dei Cavalieri 7, I-56126 Pisa, Italy

E-mail address: guido.dephilippis@sns.it

Department of Mathematics, The University of Texas at Austin, 1 University Station C1200, Austin, Texas 78712

E-mail address: figalli@math.utexas.edu 